\title{
Evolving by Maximizing the Number of Rules: Complexity Study
}

\author{
Oana Agrigoroaiei, Gabriel Ciobanu, and Andreas Resios \\ 1 Romanian Academy, Institute of Computer Science \\ Blvd. Carol I no.8, 700505 Iaşi, Romania \\ 2 "A.I.Cuza" University, Blvd. Carol I no.11, 700506 Iaşi, Romania \\ oanaag@iit.tuiasi.ro, gabriel@info.uaic.ro, andreas.resios@iit.tuiasi.ro
}

Summary. This paper presents the complexity of finding the multiset of rules in a $\mathrm{P}$ system in such a way to have a maximal number of rules applied. It is proved that the decision version of this problem is NP-complete. We study a number of subproblems obtained by considering that a rule can be applied at most once, and by considering the number of objects in the alphabet of the membrane as being fixed. When considering $\mathrm{P}$ systems with simple rules, the corresponding decision problem is in $\mathbf{P}$. When considering $\mathrm{P}$ systems having only two types of objects, and $\mathrm{P}$ systems in which a rule is applied at most once, their corresponding decision problems are NP-complete. We compare these results with those obtained for $\max O$ evolution.

\section{Introduction}

The reader is assumed to have basic knowledge of membrane computing; a good reference is [6]. Here we just mention the main biological inspiration of $\mathrm{P}$ systems, and some terminology concerning the variants of maximal parallelism we consider in this paper.

$\mathrm{P}$ systems are inspired by the structure and the functioning of the living cells. Inside the cell, several membranes define compartments where specific biochemical processes take place. Each compartment contains substances (ions, small molecules, macromolecules) and specific reactions. The substances are represented by multisets of objects, and the reactions by rules of form $u \rightarrow v$, where $u$ and $v$ are multisets of objects. The multisets are represented by strings, with the understanding that all permutations of a string represent the same multiset. We denote by $O$ the alphabet of objects, and by $R_{i}$ the set of rules associated with a compartment $i$. When such a system is evolving, the objects and the rules are chosen in a nondeterministic manner, and the rules are applied in parallel.

The most investigated way of using the rules in a $\mathrm{P}$ system is the maximal parallelism: in each membrane a multiset of rules is chosen which can be applied to the objects from that membrane and is maximal in the sense of inclusion, i.e., 
no further rule can be added such that the enlarged multiset is still applicable. We use "maxP" to refer to this evolution strategy.

Another natural idea is to apply the rules in such a way to have a maximal number of objects consumed in each membrane. This manner of evolution is denoted by " $\max O$ ". This strategy was explicitly considered in $[1,2]$, where it is proved that the problem of finding a multiset of rules consuming a maximal number of objects is NP-complete.

Yet a third idea is to apply the rules in such a way to have a maximal number of rules applied. We call this type of evolution "maxR". Note that any evolution of type $\max R$ or $\max O$ is also of type $\max P$.

The computing power of these strategies of applying a multiset of rules in membranes is studied in [3]. Specifically, P systems having multiset rewriting rules (with cooperative rules), symport/antiport rules, and active membranes are considered. The universality of the system is proved for any combination of type of system and type of evolution.

In previous papers $[1,2]$, two variants of membrane systems called simple $\mathrm{P}$ systems and maximum cooperative $\mathrm{P}$ systems are considered. They evolve at each step by consuming the maximum number of objects. The problem of distributing objects to rules in order to achieve a maximum consuming and non-deterministic evolution of simple P systems is studied in [1]; using the knapsack problem, the decision version of the resource mapping problem for simple $\mathrm{P}$ systems is proved to be NP-complete. In [2] the integer linear programming problem is used to prove that the resource mapping problem for maximum cooperative $\mathrm{P}$ systems is also NP-complete.

In this paper we study the complexity of finding a multiset of rules which evolves the membrane in the sense of $\max R$. We study a number of subproblems obtained by considering the number of objects in the alphabet of the membrane as being fixed or by considering that a rule can be applied at most once. We compare the results with those obtained for $\max O$ evolution.

\section{$2 \max R$ Complexity}

We recall a number of notations for multisets and $\mathrm{P}$ systems. We represent multisets as strings of elements over their support alphabet together with their multiplicities (for example $w=a^{2} b^{5} c$ is a multiset over $\{a, b, c, d\}$ ). The union $v+w$ of two multisets over a set $O$ is given by the sum of multiplicities for each element of $O$. We define $w(a) \in \mathbb{N}$ to be the multiplicity of $a$ in $w$. We say that $w \leq w^{\prime}$ if $w(a) \leq w^{\prime}(a)$ for each element $a$ of the multiset $w$. In this case we define $w^{\prime}-w$ to be the multiset obtained by subtracting the multiplicity in $w$ of an element from its multiplicity in $w^{\prime}$.

We use the notation $i=\overline{1, n}$ to denote $i \in\{1, \ldots, n\}$.

Definition 1. A transition $P$ system of degree $n, n \geq 1$ is a construct 


$$
\Pi=\left(O, \mu, w_{1}, \ldots, w_{n}, R_{1}, \ldots, R_{n}\right)
$$

where

- $O$ is an alphabet of objects;

- $\mu$ is a membrane structure, with the membranes labelled by natural numbers $1, \ldots, m$, in a one-to-one manner;

- $w_{i}$ are multisets over $O$ associated with the regions $1, \ldots, m$ defined by $\mu$;

- $R_{1}, \ldots, R_{m}$ are finite sets of rules associated with the membranes with labels $1, \ldots, m$; the rules have the form $u \rightarrow v$, where $u$ is a non-empty multiset of objects and $v$ a multiset over messages of the form (a, here), (a, out), $\left(a, i n_{j}\right)$.

A configuration of the system is given by the membrane structure and the multisets contained in each membrane. For a rule $r=u \rightarrow v$ we use the notations $l h s(r)=u$ and $r h s(r)=v$. These notations are extended naturally to multisets of rules: given a multiset of rules $\mathcal{R}$, the left hand side of the multiset $\operatorname{lhs}(\mathcal{R})$ is obtained by adding the left hand sides of the rules in the multiset, considered with their multiplicities.

We define the three evolution strategies as follows:

Definition 2. Let $i=\overline{1, n}$. A multiset $\mathcal{R}$ of rules over $R_{i}$ is applicable (in membrane $i)$ with respect to the multiset $w_{i}$ if $l h s(\mathcal{R}) \leq w_{i}$ and for each message $\left(a, i n_{j}\right)$ present in $r h s(\mathcal{R})$ we have that $j$ is one of the children of membrane $i$.

A multiset $\mathcal{R}$ of rules over $R_{i}$ which is applicable with respect to the multiset $w_{i}$ is called:

- maxP-applicable with respect to $w_{i}$ if there is no rule $r$ in $R_{i}$ such that $\mathcal{R}+r$ is applicable with respect to $w_{i}$;

- maxO-applicable with respect to $w_{i}$ if for any other multiset $\mathcal{R}^{\prime}$ of rules which is applicable with respect to $w_{i}$ we have that

$$
\sum_{a \in O} \operatorname{lh} s(\mathcal{R})(a) \geq \sum_{a \in O} \operatorname{lh} s\left(\mathcal{R}^{\prime}\right)(a)
$$

- maxR-applicable with respect to $w_{i}$ if for any other multiset $\mathcal{R}^{\prime}$ of rules which is applicable with respect to $w_{i}$ we have that

$$
\sum_{r \in R_{i}} \mathcal{R}(r) \geq \sum_{r \in R_{i}} \mathcal{R}^{\prime}(r) .
$$

In other words, when choosing the $\max P$ evolution strategy we only apply multisets of rules which are maximal with respect to inclusion; when choosing $\max O$ we only apply multisets of rules which are maximal with respect to the number of objects (considered with their multiplicities) in the left hand side of the multiset; when choosing $\max R$ we only apply multisets of rules which are maximal with respect to the number of rules in the multiset (considered with their multiplicities). Note that any multiset of rules which is either $\max R$ or 
max $O$-applicable is also $\max P$-applicable. $\mathrm{P}$ systems generally employ the $\max P$ evolution strategy; however, a convincing case can be made for max $O$ and $\max R$.

As it is mentioned in [3], maximizing the number of objects or the number of rules can be related to the idea of energy for controlling the evolutions of $\mathrm{P}$ systems. In the same paper, the complexity of finding the multiset of rules in a $\mathrm{P}$ system in the case of $\max R$ was presented as an open problem.

We denote by $P_{O}$ and $P_{R}$ the problems of finding a $\max O$ or $\max R$-applicable multiset of rules, with respect to a given multiset of objects $w$. We could consider similar problems for the entire system, but they are solved by splitting the problems into smaller ones, one for each membrane. So for our purposes we can just as well consider the system contains only one membrane, i.e. the degree of the $\mathrm{P}$ system is $n=1$. In other words, all multisets of rules we consider from now on are over a set of rules $R$. We use the following notations:

- $m$ is the cardinal of the alphabet $O$ and we consider the objects to be denoted by $o_{1}, \ldots, o_{m}$

- $d$ is the number of rules associated to the membrane, and the rules are denoted by $r_{1}, \ldots, r_{d}$;

- $C_{a}$ is the multiplicity of $o_{a}$ in the multiset $w$ which is in the membrane;

- $k_{i, a}$ is the multiplicity of $o_{a}$ in the left hand side of the rule $r_{i}$.

The problem $P_{O}$ can be described in the form of an integer linear programming problem as follows. Given the positive integers $m, d, k_{i, a}, C_{a}$ for $i=\overline{1, d}$ and $a=$ $\overline{1, m}$, find positive integers $x_{i}$ such that

- $\sum_{i=\overline{1, d}}\left(\sum_{a=\overline{1, m}} k_{i, a}\right) x_{i}$ is maximal;

- $\sum_{i=\overline{1, d}} x_{i} \cdot k_{i, a} \leq C_{a}$, for all $a=\overline{1, m}$.

The decision version of this problem was shown to be NP-complete in $[1,2]$. The proofs are based on the knapsack problem and integer linear programming $[4$, $5]$.

The problem $P_{R}$ can be described as follows. Given the positive integers $m, d, k_{i, a}, C_{a}$ for $i=\overline{1, d}$ and $a=\overline{1, m}$, find positive integers $x_{i}$ such that

- $\sum_{i=\overline{1, d}} x_{i}$ is maximal;

- $\sum_{i=\overline{1, d}} x_{i} \cdot k_{i, a} \leq C_{a}$, for all $a=\overline{1, m}$.

The decision version of $P_{R}$ is denoted by $D P_{R}$ : being given positive integers $m, d, t, k_{i, a}$ and $C_{a}$, find whether there exist positive integers $x_{i}$ such that

- $\sum_{i=\overline{1, d}} x_{i} \geq t$

- $\sum_{i=\overline{1, d}} x_{i} \cdot k_{i, a} \leq C_{a}$, for all $a=\overline{1, m}$.

The length of this instance of the problem can be considered to be $m+d+\max _{a, i}\left\{\log C_{a}, \log k_{i, a}\right\}$.

Proposition 1. $D P_{R}$ is $\mathbf{N P}$-complete. 
Proof. First, we prove that $D P_{R}$ is in NP. To show this we construct a Turing machine that computes the result in nondeterministic polynomial time by either accepting (output YES) or rejecting (output NO) the input string. The machine operates as follows:

1. nondeterministically assign values for $x_{i}, i=\overline{1, d}$;

2. if the assigned values verify the constraints

3. and $\sum_{i=\overline{1, d}} x_{i} \geq t$, then output YES;

4. in any other case, output NO.

It can be easily seen that the number of steps performed by the machine is polynomial with respect to the input size. Thus $D P_{R}$ is in NP.

Secondly, we construct a polynomial-time reduction from $3 C N F S A T$ to $D P_{R}$. The $3 C N F S A T$ [4] problem asks whether a formula $\phi$ given in conjunctive normal form with 3 variables per clause is satisfiable, i.e. if there exists a variable assignment which makes the formula true.

Consider a formula $\phi$ with variables $x_{1}, \ldots, x_{r}$ and clauses $c_{1}, \ldots, c_{s}$. We describe a corresponding instance of $D P_{R}$ :

- $d=2 r, m=r+s, t=r$;

- for each variable $x_{i}$ of $\phi$ we consider two variables $y_{i}$ and $z_{i}$ and an inequality $y_{i}+z_{i} \leq 1$ in the instance of $D P_{R}$;

- for each clause $c_{a}$ we consider the inequality

$$
\sum_{i=\overline{1, r}} q_{i, a} y_{i}+\sum_{i=\overline{1, r}} l_{i, a} z_{i} \leq 2
$$

such that:

- $q_{i, a}=0, l_{i, a}=1$ if the literal $x_{i}$ appears in $c_{a}$;

$-q_{i, a}=1, l_{i, a}=0$ if the literal $\neg x_{i}$ appears in $c_{a}$;

- $q_{i, a}=l_{i, a}=0$ if neither $x_{i}$ nor $\neg x_{i}$ appear in $c_{a}$.

Since we have $t=r$, the first inequality in this instance of $D P_{R}$ is $\sum_{i=\overline{1, r}} y_{i}+z_{i} \geq r$. This can be computed in polynomial time with respect to the size of the input. The idea behind the reduction is to set $x_{i}=1$ if and only if $y_{i}=1, z_{i}=0$ and $x_{i}=0$ if and only if $y_{i}=0, z_{i}=1$.

For example, consider the formula $\phi=c_{1} \wedge c_{2} \wedge c_{3} \wedge c_{4}$ with $c_{1}=x_{1} \vee \neg x_{2} \vee x_{3}$, $c_{2}=\neg x_{1} \vee \neg x_{2} \vee \neg x_{3}, c_{3}=x_{1} \vee \neg x_{2} \vee \neg x_{3}, c_{4}=\neg x_{1} \vee x_{2} \vee x_{3}$. The corresponding instance of $D P_{R}$ is: find positive integers $y_{i}, z_{i}, i=\overline{1,3}$ positive integers such that $\sum_{i=\overline{1,3}} y_{i}+z_{i} \geq 3, y_{i}+z_{i} \leq 1$, and

$$
\left\{\begin{array}{l}
z_{1}+y_{2}+z_{3} \leq 2 \\
y_{1}+y_{2}+y_{3} \leq 2 \\
z_{1}+y_{2}+y_{3} \leq 2 \\
y_{1}+z_{2}+z_{3} \leq 2
\end{array}\right.
$$


We notice that $y_{i}+z_{i}=1$, and that a solution is $y_{1}=0, y_{2}=0, z_{3}=0$, together with the corresponding values for $z_{1}, z_{2}, y_{3}$. This means that we consider the assignment $x_{1}=0, x_{2}=0, x_{3}=1$ for which the formula $\phi$ is satisfiable.

We now prove that a formula $\phi$ is satisfiable if and only if there is a vector of positive integers $\left(y_{1}, \ldots, y_{r}, z_{1}, \ldots, z_{r}\right)$ which is a solution for the above instance of $D P_{R}$. First, suppose there is a satisfying assignment for $\phi$. If $x_{i}=1$ we set $y_{i}=1, z_{i}=0$, and if $x_{i}=0$ we set $y_{i}=0, z_{i}=1$. Thus we have $y_{i}+z_{i} \leq 1$, for all $i=\overline{1, r}$, and also $\sum_{i=\overline{1, r}} y_{i}+z_{i} \geq r$. Consider now one of the inequalities

$$
\sum_{i=\overline{1, r}} q_{i, a} y_{i}+\sum_{i=\overline{1, r}} l_{i, a} z_{i} \leq 2
$$

We notice that it contains in its left hand side exactly three variables with coefficient 1 , one for each literal appearing in $C_{a}$. If the literal with value 1 in $C_{a}$ is $x_{j}$, then its corresponding variable is $z_{j}$ which is 0 . If the literal with value 1 in $C_{a}$ is $\neg x_{j}$, then its corresponding variable is $y_{j}$ which is 0 . Thus there are at most two terms equal to 1 , meaning that the inequality is satisfied.

Now suppose there is a solution $\left(y_{1}, \ldots, y_{r}, z_{1}, \ldots, z_{r}\right)$ for the $D P_{R}$ instance. Since $y_{i}+z_{i} \leq 1$ for all $i=\overline{1, r}$ and $\sum_{i=\overline{1, r}} y_{i}+z_{i} \geq r$, it follows that $y_{i}+z_{i}=1$ for all $i$. We consider the assignment $x_{i}=1$ if $y_{i}=1, z_{i}=0$ and $x_{i}=0$ if $y_{i}=0, z_{i}=1$. As previously noted, the inequality corresponding to a clause $c_{a}$ has exactly three variables, each with coefficient 1 , in its left hand side. Thus at least one of them must be equal to 0 . If that variable is $z_{j}$, it means that the literal $x_{j}$ with assignment $x_{j}=1$ appears in $C_{a}$. If that variable is $y_{j}$, it means that the literal $\neg x_{j}$ with assignment $x_{j}=0$ appears in $C_{a}$. Thus $\phi$ is satisfied.

We can also consider the problem $1 D P_{R}$ obtained from $D P_{R}$ by restricting the possible values of the variables to 0 or 1 . This corresponds to requesting that in a membrane a rule can be applied at most once. Then exactly the same reduction can be made from $3 C N F S A T$ to $1 D P_{R}$ thus placing $1 D P_{R}$ in the category of NP-complete problems.

\section{Certain Subproblems}

We denote by $D P_{R}^{k}$ the problem obtained from $D P_{R}$ by considering $m=k$ fixed. A similar notation is used for $D P_{O}^{k}$.

We start by looking at the case of a $\mathrm{P}$ system which has only simple rules, i.e. rules which have only one type of object in their right hand side. Then $D P_{R}^{1}$ describes the decision version of the problem of finding a multiset of simple rules which is max $R$-applicable: given $d, t, k_{i, 1}, C_{1}$ find $x_{i}$ such that $\sum_{i=\overline{1, d}} x_{i} \geq t$ and $\sum_{i=\overline{1, d}} x_{i} \cdot k_{i, 1} \leq C_{1}$.

Proposition 2. $D P_{R}^{1}$ is in $\boldsymbol{P}$. 
Proof. Note that all $k_{i, 1} \neq 0$ by definition, since rules always have a non-empty left hand side. Let $j$ be chosen such that $k_{j, 1}=\min _{i=\overline{1, d}}\left\{k_{i, 1}\right\}$. A solution is given by setting $x_{j}=\left[\frac{C}{k_{j, 1}}\right]$ (the integer part of $\frac{C}{k_{j, 1}}$ ) and $x_{i}=0, i \neq j$.

On a side note, consider the problem $1 D P_{R}^{1}$ obtained by restricting the possible values of $x_{i}$ to 0 or 1 . This problem is in $\mathbf{P}$, as can be seen by following this algorithm. First we renumber the coefficients $k_{i, 1}$ (together with the variables $x_{i}$ ) such that $k_{1,1} \leq k_{2,1} \leq \ldots \leq k_{d, 1}$. Then we set $s_{1}=k_{1,1}, s_{i+1}=s_{i}+k_{i+1,1}$. If $s_{d} \leq C_{1}$ then the maximum value for $\sum_{i} x_{i}$ is $d$. Otherwise, there exists an unique $j$ such that $s_{j} \leq C_{1}<s_{j+1}$. Therefore the maximum value for $\sum_{i} x_{i}$ is $j$, since however we choose $j+1$ different coefficients $k_{r_{1}, 1}, k_{r_{2}, 1}, \ldots k_{r_{j+1}, 1}$ randomly, their sum will be greater than $s_{j+1}$.

We now consider that the membrane whose $\max R$ evolution we are studying has only two types of objects, i.e. $\# O=2$. The corresponding decision problem is $D P_{R}^{2}$.

\section{Proposition 3. $D P_{R}^{2}$ is NP-complete.}

To prove this result we consider the following auxiliary problem $A P$ :

For $s, r, k$ positive integers, are there positive integers $x_{1}, \ldots, x_{s}$ such that

$$
\sum_{i=\overline{1, s}} x_{i}=r, \sum_{i=\overline{1, s}} k_{i} x_{i}=k .
$$

Note that if we restrict this problem by imposing the condition that all $x_{i} \in\{0,1\}$, then we obtain a subproblem of the subset sum problem, namely: given a set $S$ of positive integers $S=\left\{k_{i} \mid i=\overline{1, s}\right\}$, does exist a subset of $S$ with $r$ elements such that the sum of its elements equals $k$ ? This provides a strong hint that $A P$ is NPcomplete. The proof of Proposition 3 is based on constructing a polynomial-time reduction from $X 3 C$ to $A P$, and another one from $A P$ to $D P_{R}^{2}$.

Proof. First, note that both $D P_{R}^{2}$ and $A P$ are in NP. This can be easily proved by constructing a Turing machine similar to the one used in the proof of Proposition 1. Secondly, we give a a polynomial-time reduction from $X 3 C$ to $A P$. The exact cover by 3-sets problem $(X 3 C)$ asks if, given a set $X$ with $3 q$ elements and a collection $C$ of 3-element subsets of $X$, there is a subcollection $C^{\prime}$ of $C$ which is an exact cover for $X$, i.e. any element of $X$ belongs to exactly one element of $C^{\prime}$ [4].

In order to reduce $X 3 C$ to $A P$, we do the following. Let $l$ be the number of elements of $C$, and consider indexing the elements of $C$ by $c_{1}, \ldots, c_{l}$. For each $c_{i}$ we consider a variable $x_{i}$ in the $A P$ problem, thus setting $s=l$. To construct the coefficients $k_{i}$, we employ the notations $e_{i j}=\# c_{i} \cap c_{j}$ for $i, j=\overline{1, l}$, and $M=3 q+1$. We set $s=l, r=q, k_{i}=\sum_{j=\overline{1, l}} e_{i j} \cdot M^{l-j}$ and $k=\sum_{j=\overline{1, l}} 3 \cdot M^{l-j}$. For a solution $C^{\prime}$ to $X 3 C$ we set $x_{i}=1$ whenever $c_{i} \in C^{\prime}$, and $x_{i}=0$ otherwise. We prove that this yields a solution of the constructed instance of $A P$ and moreover, that any solution of the instance has $x_{i} \in\{0,1\}$ and provides a solution of $X 3 C$. 
Example. Consider the problem $X 3 C$ for $X=\{1, \ldots, 9\}$ and $c_{1}=\{1,2,3\}$, $c_{2}=\{1,3,4\}, c_{3}=\{4,5,6\}, c_{4}=\{1,6,8\}, c_{5}=\{4,7,9\}, c_{6}=\{7,8,9\}$. Then $M=10$ and the coefficients $k_{i}$ are written in base 10 such that they have a digit for each variable $x_{j}$ :

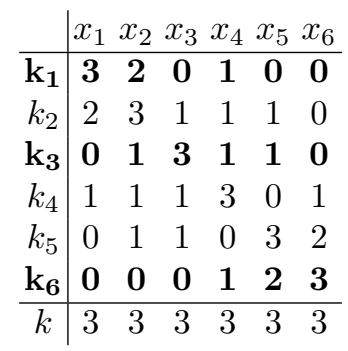

An exact cover of $X$ is $c_{1}, c_{3}, c_{6}$. Looking at this example, we see why any solution to $A P$ has all $x_{i} \in\{0,1\}$ : all coefficients have at least a digit equal to 3 and the basis $M$ is chosen such that, when adding coefficients, no carries can occur from lower digits to higher digits.

We first prove that a solution $C^{\prime}$ for $X 3 C$ provides a solution for $A P$. Let $I=\left\{i \mid c_{i} \in C^{\prime}\right\}$. Since $C^{\prime}$ is an exact cover for $X$, it follows that $I$ has $q$ elements and that $e_{i j}=0, i, j \in I, i \neq j$. Moreover, if $j \notin I$ we have that $c_{j}=c_{j} \cap\left(\cup_{i \in I} c_{i}\right)=$ $\cup\left(c_{j} \cap c_{i}\right)$, thus $\sum_{i \in I} e_{i j}=3$. Since $x_{i}=1, i \in I$ and $x_{i}=0, i \notin I$ it follows that indeed $\sum_{i=1, m} x_{i}=q$. We also have

$$
\begin{gathered}
\sum_{i=\overline{1, l}} k_{i} x_{i}=\sum_{i \in I}\left(\sum_{j=\overline{1, l}} e_{i j} M^{l-j}\right)= \\
=\sum_{i \in I}\left(e_{i i} M^{l-i}+\sum_{j \notin I} e_{i j} M^{l-j}\right)=\sum_{i \in I} 3 \cdot M^{l-i}+\sum_{j \notin I}\left(\sum_{i \in I} e_{i j}\right) M^{l-j}
\end{gathered}
$$

Using the previous observation, we obtain that the term of the second sum is $3 \cdot M^{l-j}$, thus $\sum_{i=\overline{1, m}} k_{i} x_{i}=k$.

Secondly, consider a solution $\left(x_{i}\right)_{i=\overline{1, s}}$ for the instance of $A P$ with $s, r, k_{i}, k$ as above. Let $I=\left\{i \mid x_{i}=1\right\}$. We prove that if $j \notin I$ then $x_{j}=0$ and that $e_{i j}=0$ for $i, j \in I, i \neq j$. This is sufficient to prove that $C^{\prime}=\left\{c_{i} \mid i \in I\right\}$ is an exact cover, since it follows from the above statement that $C^{\prime}$ has exactly $q$ elements and $c \cap c^{\prime}=\emptyset$ for all $c, c^{\prime} \in C^{\prime}, c \neq c^{\prime}$. We have

$$
\sum_{i=\overline{1, l}} 3 \cdot M^{l-j}=k=\sum_{i=\overline{1, l}} k_{i} x_{i}=\sum_{j=\overline{1, l}}\left(\sum_{i=\overline{1, l}} e_{i j} x_{i}\right) M^{l-j}
$$

Since $\sum_{i=\overline{1, l}} e_{i j} x_{i} \leq \sum_{i=\overline{1, l}} 3 x_{i}=3 q<M$, the two sides of equation (1) represent two decompositions in base $M$ of the same number $k$. Therefore we have $\sum_{i=\overline{1, l}} e_{i j} x_{i}=3$, for any $j=\overline{1, l}$. For $i=j$ we get $e_{i i} x_{i}=3 x_{i} \leq 3$, i.e. all $x_{i} \in$ $\{0,1\}$. Thus $3=\sum_{i \in I} e_{i j}$; considering $j \in I$ we obtain that $3=3+\sum_{i \in I, i \neq j} e_{i j}$, namely that $e_{i j}=0, i, j \in I, i \neq j$, concluding the second part of the reduction. 
We still have left to show that $A P$ reduces to $D P_{R}^{2}$. We recall the data of $D P_{R}^{2}$ : given $d, t, C_{1}, C_{2}, k_{i, 1}, k_{i, 2}$ for $i=\overline{1, d}$, do exist positive integers $x_{1}, \ldots, x_{d}$ such that

$$
\left\{\begin{array}{l}
\sum_{i=1, d} x_{i} \geq t \\
\sum_{i=1, d} k_{i, 1} x_{i} \leq C_{1} \\
\sum_{i=1, d} k_{i, 2} x_{i} \leq C_{2}
\end{array} ?\right.
$$

The reduction is as follows: let $K=\max _{i=\overline{1, d}} k_{i}$ and set $d=s, t=r, k_{i, 1}=k_{i}$, $k_{i, 2}=K-k_{i}, C_{1}=k$ and $C_{2}=K r-k$. If $x_{1}, \ldots, x_{s}$ represent a solution for the instance of $A P$, it clearly is a solution for this instance of $D P_{R}^{2}$. Reversely, if $x_{1}, \ldots, x_{s}$ represent a solution for this instance of $D P_{R}^{2}$, we add the last two inequalities of (2), obtaining $\sum_{i=\overline{1, s}} K \cdot x_{i} \leq K r$. Since $\sum_{i=\overline{1, d}} x_{i} \geq t$, we obtain that $\sum_{i=\overline{1, s}} x_{i}=r$ and also that $\sum_{i=\overline{1, s}} k_{i} x_{i}=k$.

We compare these results with those for $D P_{O}$ and its analogous subproblems. Both $D P_{R}$ and $D P_{O}$ are $\mathbf{N P}$-complete, yet we obtain significant differences when restricting to the case of $\mathrm{P}$ systems with simple rules. Namely, while $D P_{O}^{1}$ is NPcomplete, $D P_{O}^{1}$ is in $\mathbf{P}$. When we employ cooperative rules with a fixed maximum number $k$ of objects in the left hand side, the decision problems thus obtained, $D P_{O}^{k}$ and $D P_{R}^{k}$, are all NP-complete.

\section{Conclusion}

The most investigated way of applying the rules in a $\mathrm{P}$ system is the maximal parallelism ( $\max P$ case). Two other strategies of applying the rules are also possible. One strategy is to maximize the number of objects consumed in each membrane ( $\max O$ case), and the other is to maximize the number of rules applied in each membrane ( $\max R$ case).

The $\max O$ strategy was explicitly considered in [1] and [2] where it is proved that the problem of finding a multiset of rules which consume a maximal number of objects is NP-complete for both so called simple $\mathrm{P}$ systems and cooperative $\mathrm{P}$ systems.

In this paper we consider the $\max R$ strategy, and study the complexity of finding the multiset of rules in a $\mathrm{P}$ system in such a way to have a maximal number of rules applied. We prove that the decision version of this problem is NP-complete. However, in contrast to the results for $\max O$ strategy, the problem for $\mathbf{P}$ systems with simple rules is in $\mathbf{P}$.

Together with the results presented in $[1,2,3]$, this paper provides the possibility of studying complexity and computability for new classes of $\mathrm{P}$ systems. It also facilitates a complexity comparison between various classes of $\mathrm{P}$ systems.

\section{Acknowledgements}

This work has been partially supported by research grants CNCSIS IDEI 402/2007 and CNMP D1/1052/2007. 


\section{References}

1. G. Ciobanu, A. Resios. Computational Complexity of Simple P Systems. Fundamenta Informaticae vol.87, 49-59, 2008.

2. G. Ciobanu, A. Resios. Complexity of Evolution in Maximum Cooperative P Systems. Natural Computing, 2009 (to appear).

3. G. Ciobanu, S. Marcus, Gh. Păun. New Strategies of Using the Rules of a P System in a Maximal Way. Romanian Journal of Information Science and Technology vol.12, 21-37, 2009.

4. M.R. Garey, D.S. Johnson. Computers and Intractability: A Guide to the Theory of NP-Completeness. W.H.Freeman \& Co. 1979.

5. C.H. Papadimitriou, K. Steiglitz. Combinatorial Optimization: Algorithms and Complexity. Dover Publications, 1998.

6. Gh. Păun. Computing with Membranes: An Introduction, Springer, 2002. 